\title{
A Pilot Study to Evaluate an Internet-Based Cervical Cancer Screening Model Based on Self-Sampling
}

\author{
Ruifang $\mathrm{Wu}^{1,2^{*}}$, Xinfeng $\mathrm{Qu}{ }^{3}$, Hui Du${ }^{1,2}$, Zhihong Liu' ${ }^{1,2}$, Qicai Hu1, Chun Wang1,2, \\ Lijie Zhang1,2, Jinghui Zhao', Guixang Wang1,2, Jerome Leslie Belinson ${ }^{3,4}$ \\ ${ }^{1}$ Department of OB/GYN, Peking University Shenzhen Hospital, Shenzhen, China \\ ${ }^{2}$ Shenzhen Key Laboratory of Gynecological Diagnostic Technology Research, Shenzhen, China \\ ${ }^{3}$ Preventive Oncology International, Inc., Cleveland Heights, USA \\ ${ }^{4}$ Women Health Institute, Cleveland Clinic, Cleveland, USA \\ Email: *wurf100@126.com
}

Received 4 January 2016; accepted 24 May 2016; published 27 May 2016

Copyright (C) 2016 by authors and Scientific Research Publishing Inc.

This work is licensed under the Creative Commons Attribution International License (CC BY). http://creativecommons.org/licenses/by/4.0/

c) (i) Open Access

\section{Abstract}

Background: Community based cervical cancer screening models using self-collection can effectively place the identification of who is positive in the hands of the patient. The key areas we have identified as critical are 1) education, 2) notification of screening opportunities, 3) registration, 4) submission of samples, 5) receiving results with an explanation, and 6) being advised where to receive evaluation and management from qualified healthcare personnel. Methods: Our primary objectives were to create, register, and pilot a website as a public platform for cervical cancer prevention for both city and rural, individual and organizational application. We selected two sites: Shenzhen City in China as the local city (individual participant) site, and Sangzhi County in Hunan Province as the remote (group management) site. The website was reviewable to all of China, but applications for screening and registration were open only to the women from the 2 pilot sites. In the local site, the women would first sign up (obtain a password) to view the website (www.mcareu.com), and then if they desired, they could register for screening using their true name and their state ID. After an off-line confirmation of the address, the sampling kit would arrive. Results were available with additional education and guidance for care also on the website. Findings: 120,099 people nationwide visited the website in 27 months by the end of May, 2015, 1148 women from the sites registered at the website and $87.5 \%$ enrolled in the pilot trial. $72.3 \%$ of those testing positive returned for management. Virtually $100 \%$ of the registration/historical information was entered appropriately and $100 \%$ of the samples were handled properly. Interpretation: Web-based decision aids help patients through the uncertainties of healthcare. Applied to cervical cancer screening using self-collection, the effectiveness of the model specifically in re-

\footnotetext{
Corresponding author.
} 
spect to accessibility, education, registration, data input, and instructions for positive management was clearly demonstrated by this pilot as well as the potential for future diagnostics applicable to self-collection.

\section{Keywords}

\section{Cervical Cancer, Internet Screening, Self-Collection}

\section{Introduction}

Self-sampling technologies for cervical cancer screening have been studied for more than seventeen years. These studies have primarily focused on improving the accuracy of self-sampling and defining its role in screening programs [1]-[8]. Recently our group reported the development and evaluation of a community based model for cervical cancer screening based on self-sampling [9]. This model was based on the concept that population based screening programs could separate the identification of who is "test positive" (the screening) from the hospital or office based healthcare system, thereby allowing the healthcare resources (both human and financial) to be focused on the management of the positives. This model becomes especially relevant when applied to the vast populations of medically underserved. Community based screening "events" gathered self-collected samples, which were then transported to a high throughput central laboratory for processing. The results were returned to local organizers who, with the community leaders, encouraged those testing positive to attend locally arranged clinics for evaluation and management [9]-[11].

Clearly this model has many advantages primarily because it uses self-sampling for the primary screen. We now know that self-sampling is equal in accuracy to physician collected samples [8], well-accepted by patients in multiple cultures [10] [12] [13], easily adapted to medically underserved sites, and it makes massive screening events possible for populations world-wide [9]. The identification of who is positive (or sick) is an enormous consumer of healthcare resources. With this technology, it is possible for much of the logistics of screening to be assumed by the communities, or the women themselves.

Clearly some women have access to hospital based clinics or private doctors and may prefer to seek more traditional "one on one" screening and assessment. In some settings self-collection has been investigated as a possible alternative way to reach the women who simply choose not to attend available clinics [14] [15].

Although community-based screening models have enabled advanced technologies to reach the world medically underserved, they still need the involvement of the local organizers and the community leaders. Their involvement in the organization of community "events" is necessary to assure accessibility and maximize coverage.

We have learned that there are many factors that impact coverage for a population, and it is very difficult for the screening organizers to control all the variables. Along with maximizing enthusiasm for participation, having clear records of who participates is key both in the cities as well as rural communities. Often the same women get screened over and over, due to their willingness to participate and the total lack of data reporting who has been screened in the past. The organizers of screening programs need organized records to plan their programs and budgets and evaluate the outcomes of their screening efforts.

In China, even in modern cities like Shenzhen and Guangzhou, medical facility based cervical cancer screening has been conducted for more than a decade, with no impact, to date, on cervical cancer incidence and mortality. In fact it has steadily increased [16]. This fact suggests that there must be a large portion of women in cities who have never been screened and/or evaluated for a variety of reasons, or are under-screened according to current guidelines.

In our investigations of screening technologies and healthcare delivery, several issues impacting the efficiency of the screening have surfaced when millions of women are to be targeted. Millions of women need to register for screening and to be notified about their testing results. The community screening organizers who play the key roles need professionally designed programs to provide a platform to assist in planning and implementing the screening. The centralized laboratories need a platform to collect patient's identification data related to thousands of specimens and report thousands of results every day. Finally the clinics need a sharable platform to manage the positives. 
We believe that many of issues related to the screening may be more effectively managed through a webbased program. The key areas of need that we have identified are 1) education, 2) notification of screening opportunities, 3) registration, 4) submission of samples, 5) receiving results with interpretation of their meaning, and 6) being advised where to receive evaluation and management from qualified healthcare personnel. We can greatly expand the screening coverage if we have a direct link between the screening resources and the women. The Internet can serve that role. However, to construct an Internet based screening program applicable to mass population based screening, the various components need to be tested.

With these issues in mind, this pilot study was designed to test some of the key components we have identified. We also believe that successful models for cervical cancer prevention may be applied in the future to many other diseases capable of being screened or monitored with self-collected samples [17].

\section{Methods}

Our primary objectives were to create, register, and pilot a website as a public platform for cervical cancer prevention. We were interested primarily whether the targeted women could complete the registration and the management of the self-collection successfully. The website would educate women about cervical cancer prevention and encourage participation in regular screening. The site would enable women to register for screening by entering their demographic and historical details. The web-based program would then provide the women with instructions for self-collection and sample shipment to the central laboratory. After the testing was completed, the results could be found on the website. Finally, basic guidance for what the individual woman should do based on her results would be provided.

We selected Shenzhen in Guangdong Province, China, as the "local site”. This was intended to be a convenient location since the director of the screening program was based at Peking University Shenzhen Hospital. Shenzhen is an urban economically-well developed city. Sangzhi, a rural small town in Hunan Province, located more than 1000 kilometers away from Shenzhen was selected as the remote site. Our goal was to recruit 500 participants at each site. By picking these two diverse sites we hoped to learn what elements were related to the "off-line work" of the Internet based screening model that might influence the organization, coverage, participation and the call-back for positive management. In addition, the actual organizational components for managing the positive patients could be explored at the two sites.

Women living in Shenzhen could access the website through the Internet to register themselves, while women living in the remote site (Sangzhi) accessed the website with the assistance of a local coordinator who modeled the role of the community leaders as reported in our community based models [9]. Women living in both sites could voluntarily participate if they were between the ages of thirty to sixty-five, non-pregnant, and still had their cervix.

We identified Peking University Shenzhen Hospital (PUSH) in Shenzhen as the local site hospital and The People's Hospital of Sangzhi Country (PHSC) in Sangzhi County as the remote site hospital for evaluation and management of screen positive cases. The website was reviewable to all of China, but applications for screening and registration were open only to the women from the 2 pilot sites. In the local site, the women would first sign up and obtain a password to view the website (www.mcareu.com), and then if they desired, they could register for screening using their true name and their State ID.

After the woman had completed the demographic/historical data on the website (registration) she was then contacted by a phone call or text message to confirm her address sampler delivery. A positive response was followed by the sampling kit being sent to her registered address.

In the remote site, the coordinator would first sign up as an organization user of the website with authorization to register multiple women, then he/she could help the women complete registration. A sampler would then be distributed by the coordinator to the women with complete registration data. A set of criteria were set up for auto-checking the registration data as "qualified" or "unqualified”. To be categorized as "qualified" the entered data had to include all required elements: 1) Name, 2) State ID, 3) Address, 4) Cellphone number, and 5) Informed consent electronically signed by the applier. Any samples associated with "unqualified" data (one or more missing items) would not be tested.

A liquid media was provided with the sampling brush for use with the PCR based Cobas Assay (Roche, Pleasanton CA). The Cobas assay is CFDA (Chinese State FDA) approved and we had previously validated its use with self-collected specimens [18]. All the testing results were placed on the website accessible only by password, user name, and ID number. 
In Shenzhen no additional advertisement was done other than the announcement of the free screening program on the website. No off-line contact was identified. All the samplers were shipped to and samples collected from each of the participants separately via a business logistic company trained for transportation of the samples. For the remote site, the Coordinator from the People's Hospital of Sangzhi was assigned the responsibilities for registration assistance, distribution of the samplers that had previously been shipped in bulk, and collection of the samples for shipment to the central laboratory.

In Shenzhen the women could make appointments for evaluation and management in the Center of Early Diagnosis and Treatment for Cervical Carcinoma, PUSH. In Sangzhi County the women could find the date either on the website or by calling the off-line coordinator for when a team from PUSH would arrive at the People's Hospital of Sangzhi County for an evaluation and management clinic.

\section{Results}

The study was approved by the Institutional Review Board of Peking University Shenzhen Hospital. From the launch of the website at the beginning of March 2013 to the end of May 2015, 120,099 people nationwide visited the website. 1148 women within the study sites, registered for screening via the website (www.mcareu.com) and 1005/1148 (87.5\%) completed the screening. 143 women who registered but did not complete the screening were from Shenzhen. Two had incomplete registration data, eighty-four with complete data did not respond to the phone call or text to confirm their addresses for sampler delivery, and fifty-seven with complete data who responded and received the sampling kits ultimately decided not to participate (Table 1).

The HPV positive rate was $15.5 \%$ (Table 2). $72.3 \%$ of the HPV positive women have returned for management (Table 3). Proper completion of the demographic information and accurate handling of the specimens was demonstrated by $100 \%$ of the screened women (Table 4 and Table 5).

Table 1. Registration and enrollment.

\begin{tabular}{cccc}
\hline Sites & Cases registered & Case tested & $\%$ \\
\hline Shenzhen & 717 & 574 & 80.04 \\
Hunan & 431 & 431 & 100.00 \\
Total & 1148 & 1005 & 87.54 \\
\hline
\end{tabular}

Table 2. Number of positives with Cobas HR-HPV assay.

\begin{tabular}{ccccc}
\hline & N & Positives & Negatives & \% (positive) \\
\hline Shenzhen & 574 & 80 & 494 & 13.94 \\
Hunan & 431 & 75 & 356 & 17.40 \\
Total & 1005 & 155 & 848 & 15.42 \\
\hline
\end{tabular}

Table 3. Number of positives who returned for management.

\begin{tabular}{cccc}
\hline Sites & Positives & N & \% \\
\hline Shenzhen & 80 & 56 & 70.00 \\
Hunan & 75 & 56 & 74.67 \\
Total & 155 & 112 & 72.26 \\
\hline
\end{tabular}

Table 4. Proper completion of demographic information for registration ${ }^{1}$.

\begin{tabular}{cccc}
\hline Sites & Cases registered & Cases with no DI error & \% \\
\hline Shenzhen & 717 & 715 & 99.72 \\
Hunan & 431 & 431 & 100.00 \\
Total & 1148 & 1146 & 99.82 \\
\hline
\end{tabular}

${ }^{1}$ The number includes the 84 women from Shenzhen who registered with complete demographic information but did not respond to the request for mailing address. 
Table 5. Proper handling of the collected samples.

\begin{tabular}{cccc}
\hline Sites & Cases screened & Sample properly handles & $\%$ \\
\hline Shenzhen & 574 & 574 & 100 \\
Hunan & 431 & 431 & 100 \\
Total & 1005 & 1005 & 100 \\
\hline
\end{tabular}

Table 4 and Table 5 address the participants' success completing the registration procedures among those actually demonstrating an interest in pursuing the screening and in handling their sample according to the instructions on the website.

\section{Discussion}

Web-based decision aids to help patients sort out the uncertainties of healthcare and balance benefits against potential harms have been studied and reviewed in the recent literature [19] [20]. Decision aids have been specifically applied to prostate, colon, breast, and recently to lung cancer screening [21] [22]. Mobile phone notification has been studied to improve participation in screening programs [23], and a portion of the model we have proposed has been studied specifically for HPV screening using self-collected vaginal swabs [24]. Also, as noted above, the mailing of self-sampling kits has been explored as a way to reach the non-participants in organized national programs [14] [15]. In addition the Internet is extensively employed as an education medium for both patients and their healthcare providers.

In 2012 upon the completion of the CHICAPS project (Chinese Cervical Cancer Prevention Study) it was clear to us the registration process, although simplified, was a critical element that required the most attention during our training of the community leaders [9]. In addition, our experience cooperating with a central laboratory in this project also showed that data-input, as the basis for the results reporting system, was a rate-limiting step which in a mass screening program prolonged the reporting process. There were several other hurdles to be solved if the scale-up we envisioned was to be successful, so as a planning group we needed to answer several "what ifs": 1) What if a full record of screening events were needed so communities could plan and budget for future screening needs? 2) What if more basic medical facilities needed to be involved in positive management? 3) What if more than one central laboratory were to be involved in a single screening project? And 4) what if registration were to occur in other than small village units? We asked our design team if it would it be advisable to consider a web-based methodology to streamline data-input from multiple sources to potentially multiple laboratories, so as to shorten the reporting process and simplify the training of the community leaders. As these discussions continued, the registration process became incorporated into an entire web-based "front end" to the screening process. That enables the necessary information on the subjects to be "available" in the system when the specimens are shipped to the laboratory for testing. If an individual does not have personal access to the Internet, friends or village leaders and/or their administration almost certainly do.

It was with these thoughts in mind that we decided to conduct this pilot study to develop and test the feasibility of a web-based framework to comprehensibly accomplish all the elements in a screening program that are feasible to accomplish without direct physician/health provider involvement. Therefore, the model would educate women about cervical cancer prevention, and provide a mechanism for them to participate in a screening program without requiring a visit to a private doctor or a clinic. The website could instruct them how to do the screening, which would include not only collecting the samples but clear guidance for shipping the samples and receiving results. In addition, the website would provide information to help the women to better understand the meaning of their results and where to obtain skilled knowledgeable management if their test returned positive. Local medical facilities must be either available or special clinics arranged for management of the positives.

Our data demonstrates that using a web-based program we can effectively educate and register the participants in the settings we tested, and it can serve as the database for evaluation and management in both the personal surrogate site (Shenzhen) as well as the organizational screening surrogate (Sangzhi). This suggests that a professionally designed Internet based screening program can provide an effective platform to assist with community based screening. From the data we learned that the registration can be accurately completed by the participants themselves or the community leaders. We also learned the Internet system can assure that only completed demographic data will allow the testing to move forward and be linked to the test results. This we believe is a very important solution for the massive burden of registration data generated in population based screening 
programs, especially when a centralized laboratory is involved. In addition the link with the laboratory and the record of screening results provides a valuable resource for governments, NGOs, etc., to be able to determine both population and individual participation in screening programs. This we believe can be further automated to avoid the wasteful duplication of precious resources when the same individuals always appear for screening.

It is also demonstrated in this pilot project that the samplers and samples can be shipped by business logistic companies separately to the participants or in bulk to the local coordinators, suggesting that in any remote community, samplers can be shipped directly to the community leaders and distributed by them correctly.

In this project we selected the Cobas Assay because we had previously validated its accuracy with self-collected specimens [18] and it was approved by the CFDA. However, we have only recently demonstrated that Cobas can work well with filter paper-based specimen transport cards which we favor for personal use due to their ease, safety, and cost for transport [25]-[27]. Newer high-throughput and low cost assays once approved will allow their incorporation into our models [28].

The remarkable number of visitors from project and non-project areas suggests the great public interest and likely acceptance of this type of model for cervical cancer prevention.

We are encouraged by the results of this pilot project. We believe this Internet based model will significantly enhance our prior work developing models to allow today's technology to reach all the people of the world. Clearly one system or model will not work for everyone, but a menu of strategies will be needed to provide everyone with the opportunity for preventive healthcare.

\section{Conclusion}

This trial, as is the case with most pilots, is limited by having only two sites and a relatively small sample size from which to base conclusions. However, we believe that our findings are supportive of the concept and provide encouragement to expand this model. Future studies will be focused on how the Internet helps to increase the screening coverage, improve compliance with follow-up recommendations, as well as provide a structure to help standardize a model for community based screening.

\section{Funding}

A Program for Development of Common Key Technologies and Working Model for Population Based Cervical Cancer Screening: Funded by Shenzhen Municipal Science and Technical Innovation Committee (No. GJHZ 20120619145443087).

\section{References}

[1] Wright, T.C., Denny, L., Kuhn, L., Pollack, A. and Lorincz, A. (2000) DNA Testing of Self-Collected Vaginal Samples Compared with Cytologic Screening to Detect Cervical Cancer. JAMA, 283, 81-86. http://dx.doi.org/10.1001/jama.283.1.81

[2] Belinson, J.L., Qiao, Y.L., Pretorius, R.G., et al. (2001) Shanxi Province Cervical Cancer Screening Study: A CrossSectional Comparative Trial of Multiple Techniques to Detect Cervical Intraepithelial Neoplasia. Gynecologic Oncology, 83, 439-444. http://dx.doi.org/10.1006/gyno.2001.6370

[3] Belinson, J.L., Qiao, Y.L., Pretorius, R.G., et al. (2003) Shanxi Province Cervical Cancer Screening Study II: SelfSampling for High-Risk Human Papillomavirus Compared to Direct Sampling for HPV and Liquid Based Cervical Cytology. International Journal of Gynecological Cancer, 13, 1-8. http://dx.doi.org/10.1111/j.1525-1438.2003.13611.x

[4] Szarewski, A., Cadman, L., Mallett, S., et al. (2007) Human Papillomavirus Testing by Self-Sampling: Assessment of Accuracy in an Unsupervised Clinical Setting. Journal of Medical Screening, 14, 34-42. http://dx.doi.org/10.1258/096914107780154486

[5] Salmerón, J., Lazcano-Ponce, E., Lorincz, A., et al. (2003) Comparison of HPV-Based Assays with Papanicolaou Smears for Cervical Cancer Screening in Morelos State, Mexico. Cancer Causes \& Control, 14, 505-512. http://dx.doi.org/10.1023/A:1024806707399

[6] Belinson, J.L., Hu, S., Niyazi, M., et al. (2010) Prevalence of Type-Specific Human Papillomavirus in Endocervical, Upper and Lower Vaginal, Perineal, and Vaginal Self-Collected Specimens: Implications for Vaginal Self-Collection. International Journal of Gynecological Cancer, 127, 1151-1157. http://dx.doi.org/10.1002/ijc.25144

[7] Gravitt, P.E., Belinson, J.L., Salmeron, J. and Shah, K.V. (2011) Looking Ahead: A Case for HPV Testing of Self- 
Sampled Vaginal Specimens 1 as a Cervical Cancer Screening Strategy. International Journal of Cancer, 129, 517527. http://dx.doi.org/10.1002/ijc.25974

[8] Belinson, J.L., Du, H., Yang, B., et al. (2012) Improved Sensitivity of Vaginal Self-Collection and High-Risk Human Papillomavirus Testing. International Journal of Cancer, 130, 1855-1860. http://dx.doi.org/10.1002/ijc.26202

[9] Belinson, J.L., Wang, G.X., Qu, X.F., et al. (2014) The Development and Evaluation of a Community Based Model for Cervical Cancer Screening Based on Self-Sampling. Gynecologic Oncology, 132, 636-642. http://dx.doi.org/10.1016/j.ygyno.2014.01.006

[10] Levinson, K.L., Abuelo, C., Chyung, E., et al. (2013) The Peru Cervical Cancer Prevention Study (PERCAPS): Community-Based Participatory Research in Manchay, Peru. International Journal of Gynecological Cancer, 23, 141-147. http://dx.doi.org/10.1097/IGC.0b013e318275b007

[11] Abuelo, C.E., Levinson, K.L., Salmeron, J., et al. (2013) The Peru Cervical Cancer Screening Study (PERCAPS): The Design and Implementation of a Mother/Daughter Screen, Treat, and Vaccinate Program in the Peruvian Jungle. Journal of Community Health, (EPUB Ahead of Print).

[12] Tisci, S.E., Shen, Y.H., Fife, D., Goycoolea, J.M., Ma, C.P., Qiao, Y.L. and Belinson, J.L. (2003) Patient Acceptance of Self-Sampling for Human Papillomavirus in Rural China. Journal of Lower Genital Tract Disease, 7, 107-116. http://dx.doi.org/10.1097/00128360-200304000-00007

[13] Arriba, L.N., Enerson, C.L., Belinson, S., Novick, L. and Belinson, J. (2010) Mexican Cervical Cancer Screening Study II: Acceptability of Human Papillomavirus Self-Sampler. International Journal of Gynecological Cancer, 20, 1415-1423.

[14] Gök, M., Heideman, D.A., van Kemenade, F.J., et al. (2012) Offering Self-Sampling for Human Papillomavirus Testing to Non-Attendees of the Cervical Screening Program: Characteristics of the Responders. European Journal of Cancer, 48, 1799-1808. http://dx.doi.org/10.1016/j.ejca.2011.11.022

[15] Virtanen, A., Anttila, A., Luostarinen, T., Malila, N. and Nieminen, P. (2015) Improving Cervical Cancer Screening Attendance in Finland. International Journal of Cancer, 136, 677-684. http://dx.doi.org/10.1002/ijc.29176

[16] He, J., Zhao, P. and Chen, W.Q. (2012) Chinese Cancer Registry Annual Report. Military Medical Science Press, Beijing.

[17] Abramowicz, M., Zuccotti, G. and Pflomm, J.M. (2014) A Stool DNA Test (Cologuard) for Colorectal Cancer Screening. The Medical Letter on Drugs and Therapeutics, 56, 100-101.

[18] Hui, D., Wang, G., Zhang, W., et al. (2014) To Evaluate the Sensitivity of Cobas 4800 HPV Test and Seq HPV Test in Detecting Vaginal Self-Samples in Screening. 29th International Papillomavirus Conference and Public Health \& Clinical Workshops, Seattle, 21-25 August 2014, 97.

[19] Trikalinos, T.A., Wieland, L.S., Adam, G.P., Zgodic, A. and Ntzani, E.E. (2014) Decision Aids for Cancer Screening and Treatment. Report No. 15-EHC002-EF, AHRQ Comparative Effectiveness Reviews, Agency for Healthcare Research and Quality (US), Rockville.

[20] Brackett, C.D. and Kearing, S. (2015) Use of a Web-Based Survey to Facilitate Shared Decision Making for Patients Eligible for Cancer Screening. The Patient: Patient-Centered Outcomes Research, 8, 171-177.

[21] Stacy, D., Samant, R. and Bennett, C. (2008) Decision Making in Oncology: A Review of Patient Decision Aids to Support Patient Participation. CA: A Cancer Journal for Clinicians, 58, 293-304. http://dx.doi.org/10.3322/CA.2008.0006

[22] Lau, K.Y., Caverly, T.J., Cherng, S.T., Cao, P., West, M., Arenberg, D. and Meza, R. (2014) Development and Validation of a Personalized, Web-Based Decision Aid for Lung Cancer Screening Using Mixed Methods: A Study Protocol. JMIR Research Protocols, 3, e78. http://www.researchprotocols.org/2014/4/e78/ http://dx.doi.org/10.2196/resprot.4039

[23] Lee, H.Y., Koopmeiners, J.S., Rhee, T.G., Raveis, V.H. and Ahluwalia, J.S. (2014) Mobile Phone Text Messaging Intervention for Cervical Cancer Screening: Changes in Knowledge and Behavior Pre-Post Intervention. Journal of Medical Internet Research, 16, e196.

[24] Nelson, E.J., Hughes, J., Oakes, J.M., Thyagarajan, B., Pankow, J.S. and Kulasingam, S.L. (2015) Human Papillomavirus Infection in Women Who Submit Self-Collected Vaginal Swabs after Internet Recruitment. Journal of Community Health, 40, 379-386.

[25] Gustavsson, I., Sanner, K.F., Lindell, M.F., et al. (2011) Type-Specific Detection of High-Risk Human Papillomavirus (HPV) in Self-Sampled Cervicovaginal Cells Applied to FTA Elute Cartridge. Journal of Clinical Virology, 51, 255258. http://dx.doi.org/10.1016/j.jcv.2011.05.006

[26] Luo, H.X., Du, H., Maurer, K., Belinson, J.L., Wang, G.X., Liu, Z.H., Zhang, L.J., Zhou, Y.Q, Wang, C., Tang, J.L., Qu, X.F. and Wu, R.F. (2016) An Evaluation of the Cobas4800 HPV Test on Cervico-Vaginal Specimens in Liquid 
versus Solid Transport Media. PLoS ONE, 11, e0148168. http://dx.doi.org/10.1371/journal.pone.0148168

[27] Levinson, K.L., Abuelo, C., Chyung, E., et al. (2013) The Peru Cervical Cancer Prevention Study (PERCAPS): The Technology to Make Screening Accessible. Gynecologic Oncology, 129, 318-323. http://dx.doi.org/10.1016/j.ygyno.2013.01.026

[28] Yi, X., Zou, J., Xu, J., et al. (2014) Development and Validation of a New HPV Genotyping Assay Based on Next Generation Sequencing. American Journal of Clinical Pathology, 141, 796-804.

http://dx.doi.org/10.1309/AJCP9P2KJSXEKCJB 